\title{
Influência de interfases sobre a condutividade térmica efetiva de compósitos periódicos reforçados por fibras
}

\author{
Influence of interphases on the effective thermal \\ conductivity of periodic fiber-reinforced \\ composites
}

Camila de Sousa Vieira ${ }^{1}$, Severino Pereira Cavalcanti Marques ${ }^{1}$

\footnotetext{
${ }^{1}$ Programa de Pós-Graduação em Materiais, Universidade Federal de Alagoas - UFAL, CEP 57072-900, Maceió, Alagoas, Brasil.

e-mail: milavieira@gmail.com, smarques@lccv.ufal.br
}

\begin{abstract}
RESUMO
Os materiais compósitos comumente apresentam delgadas regiões ou interfases localizadas entre a matriz e as inclusões. Estas interfases têm influência no comportamento efetivo do compósito. Em diversas aplicações, os compósitos estão sujeitos a variações significativas de temperatura e, consequentemente, a condutividade térmica efetiva desses materiais se torna parâmetro essencial para compreensão do seu comportamento. $\mathrm{O}$ objetivo desse trabalho é determinar a influência de interfases na condutividade térmica efetiva de compósitos reforçados com fibras unidirecionais distribuídas periodicamente, utilizando a formulação paramétrica da Teoria de Volumes Finitos. Para isso, são avaliados os efeitos da espessura e condutividade térmica da interfase, assim como do raio da fibra e de sua fração volumétrica. Compósitos com fibras naturais são apresentados dentre os exemplos analisados. Os resultados obtidos com a Teoria de Volumes Finitos são confrontados com outros encontrados por métodos numéricos ou experimentos. Desse modo, observa-se que a interfase possui mais influência quanto maior for a fração volumétrica de fibras e quanto menor o raio das mesmas. Quanto mais espessa a interfase, mais suas propriedades influenciam a condutividade térmica efetiva do compósito, quando a fração volumétrica de fibras é mantida constante. Conclui-se que os efeitos de interfase sobre o comportamento térmico de compósitos são de extrema importância e, consequentemente, eles precisam ser considerados para proporcionar predições consistentes e realísticas.
\end{abstract}

Palavras-chave: compósitos periódicos, condutividade térmica efetiva, interfase, teoria de volumes finitos.

\section{ABSTRACT}

Composite materials commonly present thin regions or interphases located between the matrix and inclusions. These interphases influence the effective behavior of the composite. In many applications, composite materials are submitted to significant temperature variations and, consequently, the effective thermal conductivity of these materials becomes essential to understand their behavior. The purpose of this paper is to determine the influence of interphases on the effective thermal conductivity of periodic fiber reinforced composites, by using the parametric finite-volume theory. To this end, the effects of the thickness and thermal conductivity of the interphase are evaluated, as well as the fiber radius and its volumetric fraction. Composites with natural fibers are included in the analyzed examples. The results obtained with finite-volume theory are compared with other results found by numerical methods or experiments. Thus, it is noted that the interphase has a more pronounced influence on the composite effective thermal conductivity for larger fiber volume fraction and smaller fiber radius. The larger the interphase thickness, more its properties influence the effective thermal conductivity, when the fiber volume fraction is fixed. In conclusion, the interphase effects on the behavior of composite materials are extremely important and, consequently, they need to be considered to allow consistent and realistic predictions.

Keywords: periodic composites, effective thermal conductivity, interphase, finite-volume theory.

\section{INTRODUÇÃO}


As atuais demandas do setor de materiais exigem que os mesmos possuam características e desempenho cada vez mais avançados e, neste contexto, os compósitos têm encontrado um reconhecido lugar de destaque. Tais materiais são constituídos por uma matriz envolvendo inclusões que, em geral, são partículas ou fibras. Em casos especiais, estas inclusões podem ser poros.

Frequentemente, os compósitos apresentam delgadas regiões, chamadas interfases, localizadas entre a matriz e as inclusões. Estas interfases constituem zonas de transição que podem ser geradas durante o processo de fabricação do material devido às interações químicas entre a matriz e as inclusões ou ser inseridas estrategicamente no material visando aperfeiçoar seu desempenho com relação a determinadas propriedades desejadas. A influência destas interfases sobre o comportamento efetivo do compósito depende de vários fatores físicos e geométricos, incluindo a fração volumétrica e as dimensões de suas inclusões.

Em diversas aplicações, os materiais compósitos estão sujeitos a variações significativas de temperatura, como escudos térmicos [1] e dispositivos eletrônicos [2], tornando essencial o conhecimento do processo de condução de calor nesses materiais. A condução de calor nos compósitos depende fundamentalmente de sua condutividade térmica efetiva. Para compósitos poliméricos, os principais fatores que afetam essa propriedade são a condutividade do reforço, sua fração volumétrica e sua geometria, além da adesão entre a matriz e o reforço e das propriedades da interfase [3]. Interfases altamente condutivas podem aumentar significativamente a condutividade efetiva do compósito quando o tamanho da inclusão diminui, demonstrando significante efeito de tamanho [4]. A condutividade térmica efetiva de compósitos considerando a presença de interfases pode ser determinada por métodos analíticos [5-7] e numéricos, tais como o Método dos Elementos Finitos [8-10] e a Teoria de Volumes Finitos [11].

O objetivo deste artigo é avaliar a influência de interfases na condutividade térmica efetiva de compósitos reforçados com fibras unidirecionais distribuídas periodicamente, utilizando a formulação paramétrica da Teoria de Volumes Finitos, desenvolvida por CAVALCANTI et al. [12]. A célula unitária que representa o material é admitida como formada por três fases constituintes: inclusão, matriz e interfase. São avaliados os efeitos da espessura e condutividade térmica da interfase, assim como do raio da fibra e de sua fração volumétrica. Dentre os exemplos analisados, destacam-se casos de compósitos com fibras naturais. Os resultados obtidos demonstram a influência dessas variáveis na condutividade efetiva, evidenciando a importância da consideração de interfases na predição das propriedades efetivas dos materiais compósitos.

\section{Determinação da condutividade térmica efetiva através da versão paramétrica da teoria de volumes finitos}

Nesta seção é apresentada de forma sucinta a formulação da versão paramétrica da Teoria de Volumes Finitos (TVF), usada para determinar numericamente a condutividade térmica efetiva de um compósito com microestrutura periódica. Maiores detalhes da formulação completa podem ser encontrados em [11].

Neste trabalho, considera-se que o compósito é reforçado por fibras unidirecionais distribuídas periodicamente. Devido a esta característica, pode-se considerar que o elemento de volume representativo (EVR) é formado pelo agrupamento de células unitárias de repetição (CUR) como exemplificado na Figura 1.

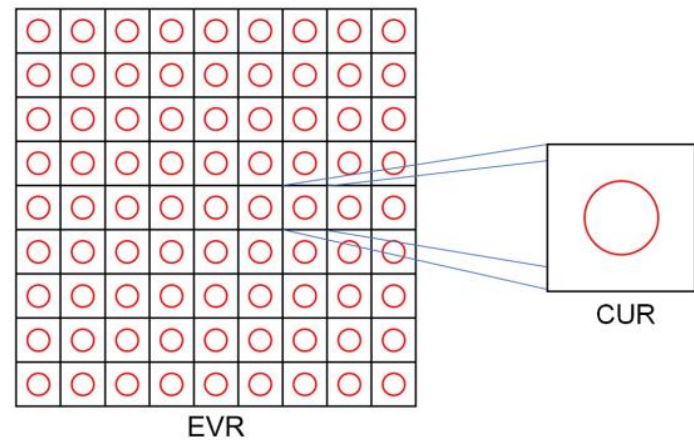

Figura 1: Elemento de Volume Representativo e Célula Unitária de Repetição.

Com base em considerações da teoria micromecânica, pode-se concluir que a resposta efetiva do EVR sob condições de contorno homogênea em temperatura coincide com a resposta de uma CUR sob adequadas condições de contorno periódicas, como mostrado em ESCARPINI FILHO e MARQUES [11]. Dessa forma, o problema de homogeneização de um compósito periódico pode ser tratado considerando apenas uma célula 
unitária de repetição.

Para determinar a condutividade térmica efetiva do compósito, a CUR é discretizada usando subvolumes quadrilaterais. Na formulação paramétrica da teoria de volumes finitos é feito o mapeamento de um quadrado de referência em um sistema de coordenadas paramétricas $(\eta-\xi)$ para um subvolume quadrilateral no plano cartesiano $\left(y_{2}-y_{3}\right)$ [12]. Na Figura 2 é mostrado um exemplo de célula unitária discretizada em subvolumes quadrilaterais. Nota-se a presença de três fases, quais sejam, a matriz, a interfase e a fibra. Esta figura apresenta a fibra envolvida por uma fina interfase (região vermelha) contornada pela matriz.

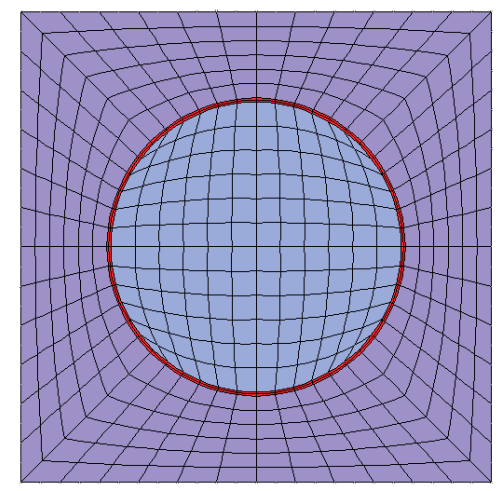

Figura 2: Célula unitária de repetição discretizada em subvolumes quadrilaterais.

\subsection{Condutividade térmica de um subvolume}

O campo de temperatura da célula unitária de repetição, com volume $\Omega$ e superfície $\Gamma$, pode ser representado na seguinte forma

$$
T\left(y_{i}\right)=T^{0}\left(x_{i}\right)+\tilde{T}\left(y_{i}\right)
$$

onde $T^{0}$ e $\tilde{T}$ são, respectivamente, as parcelas macroscópica e flutuante da temperatura. As coordenadas locais $y_{i}$ são usadas na escala da CUR, enquanto as coordenadas globais $x_{i}$ são usadas na escala do EVR.

Considerando um subvolume homogêneo e a Equação (1), as componentes do fluxo de calor são definidas pela Equação (2), onde $k_{i j}$ são as condutividades térmicas do material e $G_{j}^{0}$ são as componentes do gradiente de temperatura macroscópico.

$$
q_{i}=-k_{i j} \frac{\partial T}{\partial y_{j}}=-k_{i j} G_{j}^{0}-k_{i j} \frac{\partial \tilde{T}}{\partial y_{j}}
$$

O campo de temperatura flutuante é aproximado por uma expansão polinomial de segunda ordem de Legendre usando as coordenadas paramétricas:

$$
\widetilde{T}(\eta, \xi)=\widetilde{T}_{00}+\eta \widetilde{T}_{10}+\xi \widetilde{T}_{01}+\frac{1}{2}\left(3 \eta^{2}-1\right) \widetilde{T}_{20}+\frac{1}{2}\left(3 \xi^{2}-1\right) \widetilde{T}_{02}
$$

onde $\widetilde{T}_{m n}$ são coeficientes desconhecidos. Considera-se que o campo de temperatura é independente de $y_{1}$.

A partir da Equação (3) podem ser calculados os valores médios da temperatura flutuante, assim como do gradiente de temperatura, nas faces dos subvolumes. Os gradientes médios de temperatura em uma face $p$ do subvolume no sistema de referência podem ser relacionados com o sistema de coordenadas da microestrutura real através da média volumétrica da matriz Jacobiana (Equação 4).

$$
\left[\begin{array}{l}
\left\langle\frac{\partial \tilde{T}}{\partial y_{2}}\right\rangle \\
\left\langle\frac{\partial \tilde{T}}{\partial y_{3}}\right\rangle
\end{array}\right]^{(p)}=\left[\hat{J} \cdot\left[\begin{array}{l}
\left\langle\frac{\partial \tilde{T}}{\partial \eta}\right\rangle \\
\left\langle\frac{\partial \tilde{T}}{\partial \xi}\right\rangle
\end{array}\right]^{(p)}\right.
$$


O valor médio do fluxo de calor nas faces do subvolume é obtido substituindo-se a Equação (4) na (2):

$$
\{\hat{q}\}^{(p)}=[\bar{k}]\left\{G^{0}\right\}+[\bar{k}]\left[\hat{J}-\left[\begin{array}{l}
\left\langle\frac{\partial \tilde{T}}{\partial \eta}\right\rangle \\
\left\langle\frac{\partial \tilde{T}}{\partial \xi}\right\rangle
\end{array}\right]^{(p)} \quad \therefore[\bar{k}]=-\left[\begin{array}{ll}
k_{22} & k_{23} \\
k_{23} & k_{33}
\end{array}\right]\right.
$$

O fluxo de calor médio pode ser projetado numa direção normal à face $p$, utilizando um vetor normal unitário $\{n\}^{(p)}=\left[n_{2}, n_{3}\right]^{(p)}$ :

$$
\left[\begin{array}{l}
\hat{q}_{n}^{(1)} \\
\hat{q}_{n}^{(2)} \\
\hat{q}_{n}^{(3)} \\
\hat{q}_{n}^{(4)}
\end{array}\right]=\left[\begin{array}{l}
\{n\}^{(1)} \\
\{n\}^{(2)} \\
\{n\}^{(3)} \\
\{n\}^{(4)}
\end{array}\right][\bar{k}]\left\{G^{0}\right\}+\left[\begin{array}{l}
\{n\}^{(1)} \\
\{n\}^{(2)} \\
\{n\}^{(3)} \\
\{n\}^{(4)}
\end{array}\right][\bar{k}][\hat{J}]\left[\begin{array}{c}
\left\langle\frac{\partial \tilde{T}}{\partial \eta}\right\rangle \\
\left\langle\frac{\partial \tilde{T}}{\partial \xi}\right\rangle
\end{array}\right]^{(p)}
$$

\subsection{Matriz de condutividade térmica local de um subvolume}

A matriz de condutividade térmica do subvolume relaciona a temperatura média flutuante com o fluxo de calor médio em cada face do subvolume. Os gradientes médios de temperatura em tais faces, calculados a partir da Equação (3), podem ser incluídos na Equação (6). Desta forma, obtém-se a seguinte expressão:

$$
\left.\left[\begin{array}{l}
\hat{q}_{n}^{(1)} \\
\hat{q}_{n}^{(2)} \\
\hat{q}_{n}^{(3)} \\
\hat{q}_{n}^{(4)}
\end{array}\right]=\left[\begin{array}{l}
\{n\}^{(1)} \\
\{n\}^{(2)} \\
\{n\}^{(3)} \\
\{n\}^{(4)}
\end{array}\right][\bar{k}]\left\{G^{0}\right\}+\left[\begin{array}{c}
\{v\}^{(1)} \\
\{v\}^{(2)} \\
\{v\}^{(3)} \\
\{v\}^{(4)}
\end{array}\right]\left[\begin{array}{c}
\tilde{T}_{10} \\
\tilde{T}_{01} \\
\tilde{T}_{20} \\
\tilde{T}_{02}
\end{array}\right] \quad \therefore\{v\}^{(p)}=\{n\}^{(p)}[\bar{k}][\hat{J}] a\right]^{(p)}
$$

A matriz $[a]$ para cada face é obtida pelas seguintes expressões:

$$
[a]^{(1,3)}=\left[\begin{array}{cccc}
1 & 0 & 0 & 0 \\
0 & 1 & 0 & \mp 3
\end{array}\right] \quad[a]^{(2,4)}=\left[\begin{array}{cccc}
1 & 0 & \pm 3 & 0 \\
0 & 1 & 0 & 0
\end{array}\right]
$$

Para determinar os coeficientes desconhecidos $\tilde{T}_{m n}$, primeiramente calcula-se as derivadas segundas do campo de temperatura flutuante (Equação 3). Em seguida, utiliza-se a inversa da matriz Jacobiana para obter essas derivadas com relação às coordenadas $\left(y_{2}, y_{3}\right)$. Incluindo essas derivadas na equação de balanço de fluxo de calor e considerando a equação resultante em conjunto com as temperaturas médias flutuantes nas faces, são obtidos os coeficientes $\tilde{T}_{00}$. Considerando as temperaturas médias flutuantes nas faces em conjunto com os valores de $\widetilde{T}_{00}$ e que o material é isotrópico, $k_{22}=k_{33}=k$ e $k_{23}=k_{32}=0$, obtém-se a seguinte expressão:

$$
\left[\begin{array}{c}
\tilde{T}_{10} \\
\tilde{T}_{01} \\
\tilde{T}_{20} \\
\tilde{T}_{02}
\end{array}\right]=\left[\begin{array}{cccc}
0 & \frac{1}{2} & 0 & -\frac{1}{2} \\
-\frac{1}{2} & 0 & \frac{1}{2} & 0 \\
-\omega & \frac{1}{2}-\lambda & -\omega & \frac{1}{2}-\lambda \\
\frac{1}{2}-\omega & -\lambda & \frac{1}{2}-\omega & -\lambda
\end{array}\right]\left[\begin{array}{c}
\hat{\tilde{T}}^{(1)} \\
\hat{\tilde{T}}^{(2)} \\
\hat{\tilde{T}}^{(3)} \\
\tilde{\tilde{T}}^{(4)}
\end{array}\right]=[B]\left[\begin{array}{c}
\hat{\tilde{T}}^{(1)} \\
\hat{\tilde{T}}^{(2)} \\
\hat{\tilde{T}}^{(3)} \\
\hat{\tilde{T}}^{(4)}
\end{array}\right]
$$

Os coeficientes $\lambda$ e $\omega$ são calculados com as expressões a seguir: 


$$
\lambda=\frac{\hat{J}_{11}^{2}+\hat{J}_{21}^{2}}{2\left(\hat{J}_{11}^{2}+\hat{J}_{12}^{2}+\hat{J}_{21}^{2}+\hat{J}_{22}^{2}\right)} \quad \omega=\frac{\hat{J}_{12}^{2}+\hat{J}_{22}^{2}}{2\left(\hat{J}_{11}^{2}+\hat{J}_{12}^{2}+\hat{J}_{21}^{2}+\hat{J}_{22}^{2}\right)}
$$

Substituindo a Equação (9) na (7) obtém-se a seguinte expressão:

$$
\left\{\hat{q}_{n}\right\}^{(p)}=[N][\bar{k}]\left\{G^{0}\right\}+\left[K_{L}\right] \hat{\tilde{T}}
$$

Por fim, a Equação (12) é usada para calcular a matriz de condutividade térmica local.

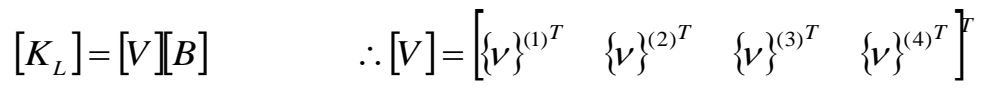

\subsection{Matriz de condutividade térmica global}

Uma vez obtida a matriz de condutividade térmica local dos subvolumes, monta-se um sistema de equações globais que inclui a matriz de condutividade térmica global $\left[K_{G}\right]$. Na construção da matriz $\left[K_{G}\right]$, as matrizes locais $\left[K_{L}\right]$ são agrupadas de acordo com as condições de compatibilidade de temperatura flutuante e fluxo de calor normal médios nas faces dos subvolumes.

As faces de cada subvolume possuem uma numeração global, sendo que as faces internas de subvolumes adjacentes possuem o mesmo número. As faces externas dos subvolumes localizados no contorno da CUR são numeradas de acordo com as condições de periodicidade. Aplicando condições de contorno periódicas, o seguinte sistema de equações globais é montado:

$$
\left[K_{G}\right]\left\{\tilde{T}_{G}\right\}=\left\{Q_{G}^{0}\right\}
$$

O vetor $\left\{\tilde{T}_{G}\right\}$ é composto pelas temperaturas flutuantes médias desconhecidas das faces dos subvolumes, enquanto o vetor $\left\{Q_{G}^{0}\right\}$ é constituído pelos fluxos normais médios macroscópicos nas interfaces dos subvolumes adjacentes. Devido à singularidade da matriz $K_{G}$, a solução do sistema (13) é obtida impondo-se temperaturas flutuantes médias nulas nas faces externas dos subvolumes dos cantos.

\subsection{Matriz de condutividade térmica homogeneizada}

A lei de Fourier para o compósito relaciona o fluxo de calor efetivo $Q^{*}$ com o gradiente de temperatura macroscópico $\left\{G^{0}\right\}$ :

$$
\left\{Q^{*}\right\}=-\left[K^{*}\right]\left\{G^{0}\right\}
$$

onde $\left[K^{*}\right]$ é matriz de condutividade térmica efetiva. Com base na discretização da célula unitária, o fluxo de calor efetivo pode ser determinado pela seguinte expressão:

$$
\left\{Q^{*}\right\}=-\sum_{r=1}^{N_{m}} k_{m} \bar{\nu}_{m}^{(r)}\left\{\hat{G}_{m}\right\}^{(r)}-\sum_{q=1}^{N_{f}} k_{f} \bar{\nu}_{f}^{(q)}\left\{\hat{G}_{f}\right\}^{(q)}-\sum_{s=1}^{N_{I}} k_{I} \bar{\nu}_{I}^{(s)}\left\{\hat{G}_{I}\right\}^{(s)}
$$

onde $N_{m}, N_{f}$ e $N_{I}$ indicam os números de subvolumes utilizados na discretização do domínio da matriz, da fibra e da interfase, respectivamente. As frações volumétricas dos subvolumes da matriz, fibra e interfase são representadas, respectivamente, por $\bar{v}_{m}^{(k)}, \bar{v}_{f}^{(k)}$ e $\bar{v}_{I}^{(k)}$.

Os gradientes médios de temperatura para os subvolumes de matriz $\hat{G}_{m}{ }^{(k)}$, fibra $\hat{G}_{f}^{(k)}$ e interfase $\hat{G}_{I}^{(k)}$ são calculados com o auxílio de matrizes de concentração de gradiente de temperatura no subvolume:

$$
\{\hat{G}\}^{(k)}=[H]^{(k)}\left\{G^{0}\right\}
$$

Por fim, substituindo a Equação 15 na 14, juntamente com a expressão 16, obtém-se a matriz de condutividade térmica homogeneizada:

$$
\left[K^{*}\right]=\sum_{r=1}^{N_{m}} k_{m} \bar{\nu}_{m}^{(r)}\left[H_{m}\right]^{(r)}+\sum_{q=1}^{N_{f}} k_{f} \bar{\nu}_{f}^{(q)}\left[H_{f}\right]^{(q)}+\sum_{s=1}^{N_{I}} k_{I} \bar{\nu}_{I}^{(s)}\left[H_{I}\right]^{(s)}
$$


As matrizes de concentração de gradiente de temperatura $[H]^{(k)}$, quadradas de ordem 2, são calculadas por meio da aplicação de gradientes macroscópicos de temperatura elementares. A primeira coluna da matriz é calculada com a aplicação de $\left\{G^{0}\right\}=[1,0]^{\mathrm{T}}$, enquanto a segunda coluna é obtida com $\left\{G^{0}\right\}=[0,1]^{\mathrm{T}}$. Assim, impondo valores para $\left\{G^{0}\right\}$, monta-se $\left\{Q_{G}^{0}\right\}$ que é o vetor dos valores conhecidos do sistema global. A solução do sistema fornece os valores de temperatura flutuante em todas as faces que, por sua vez, permitem o cálculo dos gradientes médios de temperatura. Aplicando tais resultados na Equação (16), obtém-se uma coluna da matriz $[H]^{(k)}$.

\section{EXEMPLOS}

Para a obtenção da condutividade térmica efetiva nos exemplos apresentados nesta seção, foram utilizadas células unitárias de repetição com três fases, as quais são: matriz, inclusão e interfase. A discretização da CUR é mostrada na Figura 2.

\subsection{Influência da fração volumétrica de fibras}

O objetivo deste exemplo é verificar a influência da fração volumétrica de fibras na condutividade térmica efetiva considerando a presença de uma interfase de $20 \mathrm{~nm}$ de espessura. Para isso, variou-se a fração volumétrica de $5 \%$ a $60 \%$. A condutividade térmica da matriz é igual a $0,3 \mathrm{~W} /(\mathrm{mK})$, a fibra tem $10 \mu \mathrm{m}$ de raio e sua condutividade térmica vale $300 \mathrm{~W} /(\mathrm{mK})$. No primeiro caso avaliado, a interfase possui condutividade $\left(\mathrm{k}_{\mathrm{i}}\right)$ menor do que a matriz $\left(\mathrm{k}_{\mathrm{m}}\right)$, apresentando os seguintes valores: $0,1 \mathrm{k}_{\mathrm{m}}, 0,01 \mathrm{k}_{\mathrm{m}} \mathrm{e} 0,001 \mathrm{k}_{\mathrm{m}}$. No segundo, a condutividade da interfase possui alta condutividade e são considerados os seguintes valores: $10 \mathrm{k}_{\mathrm{m}}, 100 \mathrm{k}_{\mathrm{m}} \mathrm{e}$ $1000 \mathrm{k}_{\mathrm{m}}$.

A condutividade térmica efetiva dos compósitos cujas interfases são pouco condutivas é apresentada na Figura 3, enquanto os compósitos com interfases altamente condutivas têm sua condutividade efetiva mostrada na Figura 4. Como mostrado na Figura 3, a condutividade térmica efetiva aumenta juntamente com a fração volumétrica para os casos em que $\mathrm{k}_{\mathrm{i}}$ é igual a $0,1 \mathrm{k}_{\mathrm{m}}$ e $0,01 \mathrm{k}_{\mathrm{m}}$. Mesmo com a presença de uma interfase pouco condutiva, a alta condutividade térmica da fibra permitiu um aumento na condutividade efetiva. Apesar do aumento da condutividade efetiva, nota-se a influência da interfase no fato de $\mathrm{k}^{*}$ crescer de forma menos acentuada para $\mathrm{k}_{\mathrm{i}}$ igual a $0,01 \mathrm{k}_{\mathrm{m}}$. Na média, a condutividade efetiva aumentou $17 \%$ menos quando $\mathrm{k}_{\mathrm{i}}$ passa de $0,1 \mathrm{k}_{\mathrm{m}}$ para $0,01 \mathrm{k}_{\mathrm{m}}$.

Para o caso de interfase com condutividade extremamente baixa, $\mathrm{k}_{\mathrm{i}}$ igual a $0,001 \mathrm{k}_{\mathrm{m}}$, a condutividade efetiva diminuiu mesmo com a presença de fibras altamente condutivas. Percebe-se que quanto maior a fração volumétrica de fibras, maior é a influência da interfase na condutividade efetiva do compósito.

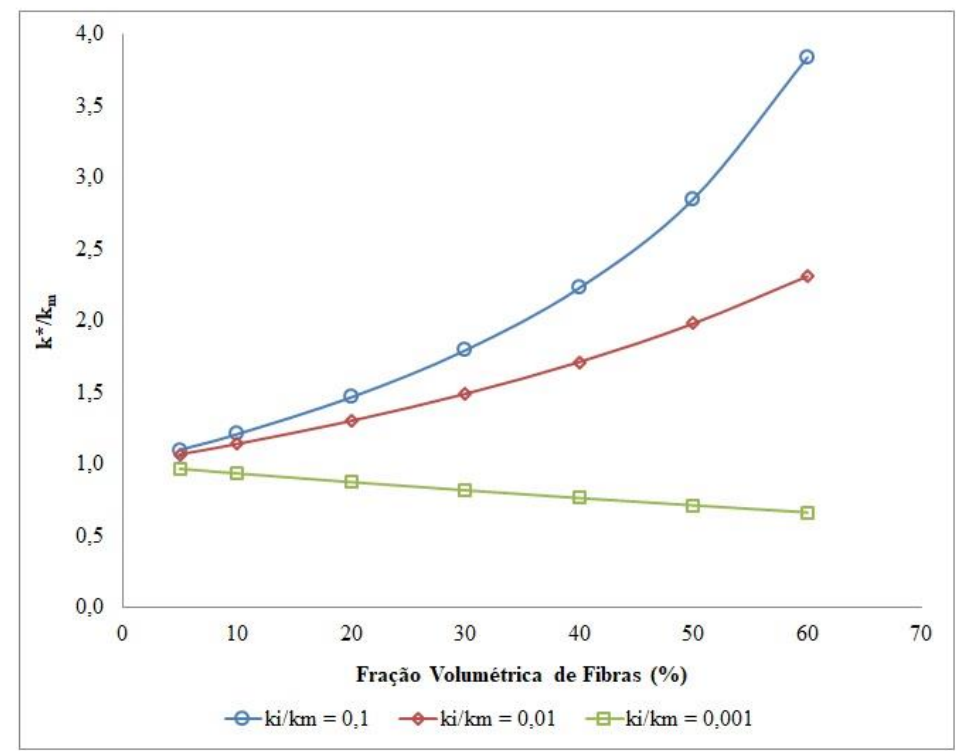

Figura 3: Condutividade térmica efetiva de compósitos com interfases de baixa condutividade.

Neste exemplo, o raio da fibra é constante, então o crescimento da fração volumétrica implica em aumento da proporção interfase/matriz. Logo, a presença de interfases com condutividade maior do que a ma- 
triz causa o aumento da condutividade térmica efetiva em conjunto com a fração volumétrica, como mostrado na Figura 4. Para o caso de interfase altamente condutiva, percebe-se neste caso que a condutividade efetiva independe da relação $\mathrm{k}_{\mathrm{i}} / \mathrm{k}_{\mathrm{m}}$.

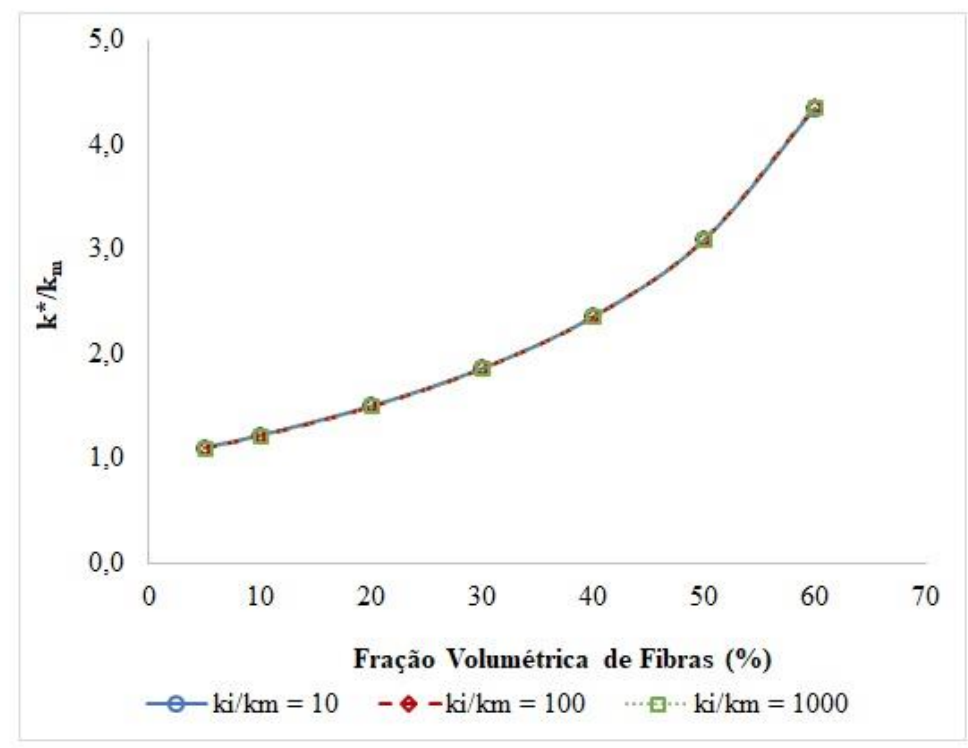

Figura 4: Condutividade térmica efetiva de compósitos com interfases de alta condutividade.

\subsection{Efeito do tamanho da inclusão}

Neste exemplo é avaliada a influência do tamanho da inclusão na condutividade térmica efetiva considerando a presença de uma interfase com $20 \mathrm{~nm}$ de espessura.

A condutividade térmica da fibra é igual a 3,5 W/(mK), o raio da mesma varia de $0,25 \mu \mathrm{m}$ a $10 \mu \mathrm{m}$ e $30 \%$ de fração volumétrica. Por sua vez, a condutividade térmica da matriz vale $0,19 \mathrm{~W} /(\mathrm{mK})$. São consideradas dois casos para a interfase: altamente condutiva, onde a condutividade térmica é igual a $100 \mathrm{k}_{\mathrm{m}} \mathrm{W} /(\mathrm{mK})$ e pobremente condutiva, cuja condutividade vale $0,01 \mathrm{k}_{\mathrm{m}} \mathrm{W} /(\mathrm{mK})$.

Na Figura 5 é mostrada a condutividade térmica efetiva para o compósito com interfase de baixa condutividade, assim como para o compósito sem interfase. Percebe-se que, quando não se considera a presença de interfase, o raio da fibra não tem influência na condutividade efetiva. Por outro lado, observa-se claramente o efeito de tamanho da fibra quando se considera a presença da interfase. No caso em estudo, a condutividade efetiva cresce na medida em que aumenta o raio da fibra, pois a interfase possui condutividade menor que a matriz. Isso acontece porque, mantendo-se a fração volumétrica constante, quanto maior o raio da fibra menor o volume total de interfase, diminuindo sua influência na condutividade efetiva. $\mathrm{O}$ efeito da dependência do tamanho da inclusão na condutividade térmica efetiva é conhecido como efeito Kapitza [13]. 


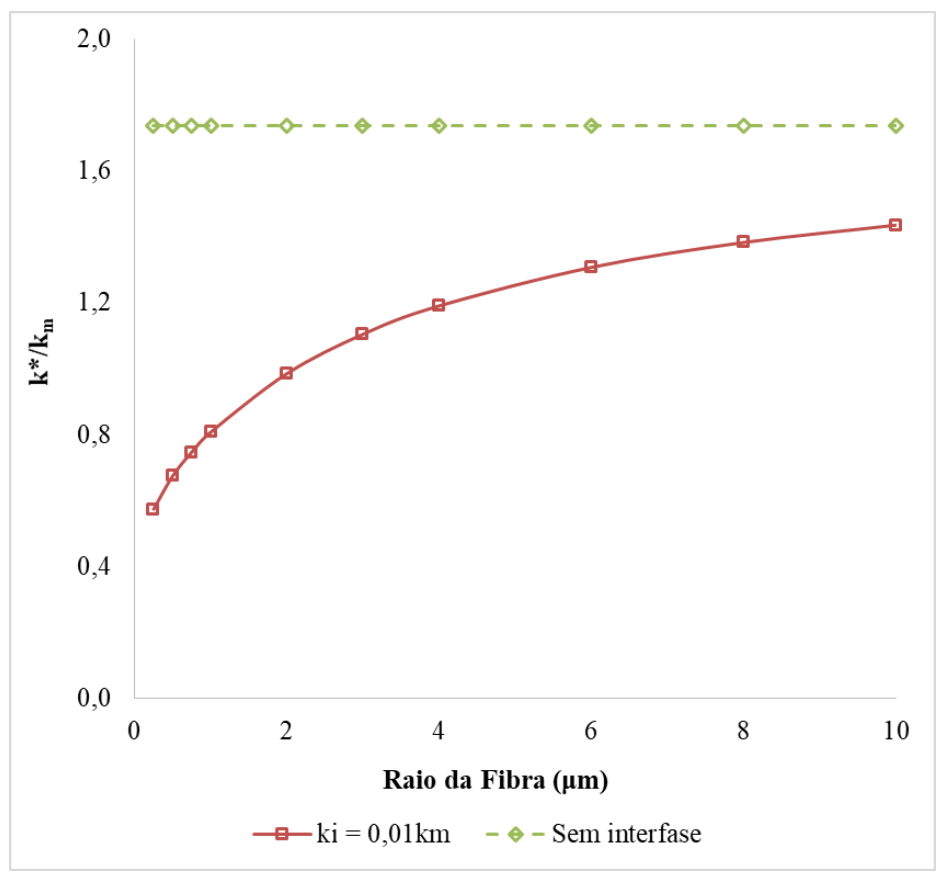

Figura 5: Influência do raio da fibra com interfase de baixa condutividade.

Na Figura 6 é apresentada a condutividade térmica efetiva para o compósito com interfase altamente condutiva e para o compósito sem interfase. $\mathrm{O}$ efeito de tamanho, citado anteriormente, também é notado neste caso, onde percebe-se que a condutividade efetiva diminui com o aumento do raio da fibra. A condutividade efetiva do compósito com interfase altamente condutiva se aproxima mais rapidamente da condutividade efetiva do compósito sem interfase, na medida em que cresce o raio, do que no caso onde o compósito tem interfase com baixa condutividade.

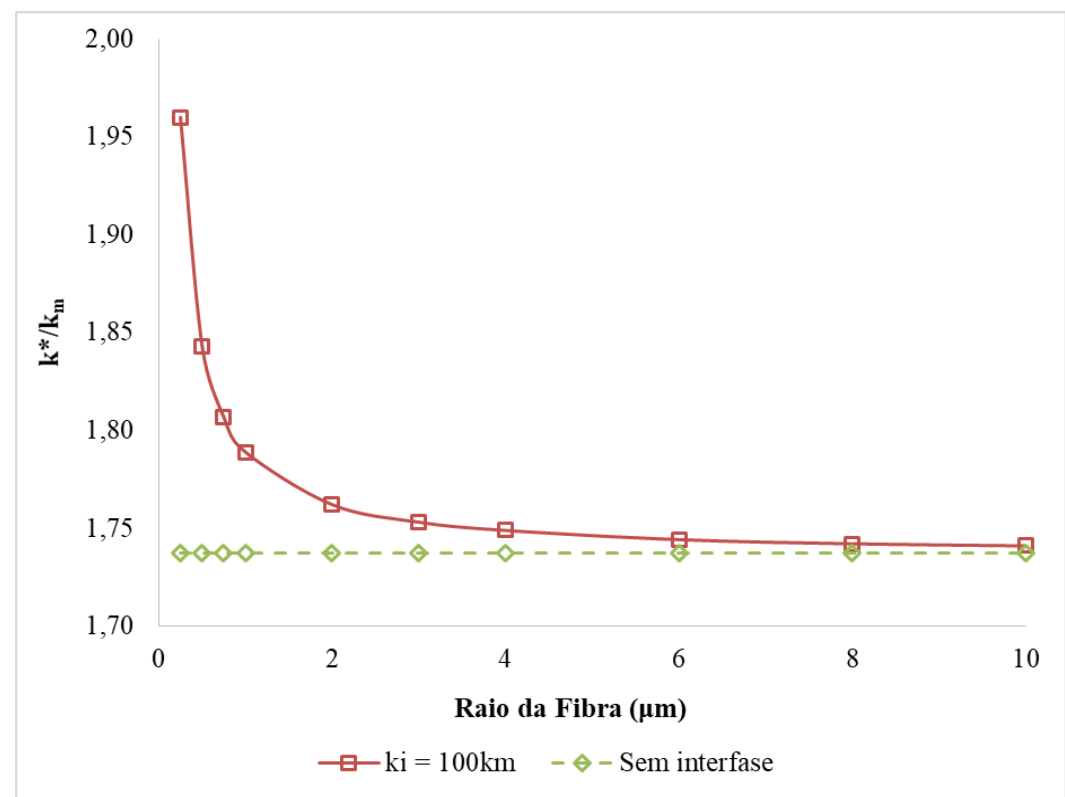

Figura 6: Influência do raio da fibra com interfase de alta condutividade.

\subsection{Efeito da espessura da interfase}

O objetivo deste exemplo é investigar a influência da espessura da interfase na condutividade térmica efetiva de um compósito com matriz epóxi. Foram considerados dois tipos de fibra, carbono e vidro, cujas propriedades são mostradas na Tabela 1. A fração volumétrica de fibras é $10 \%$ e a condutividade térmica da matriz é 
igual a $0,19 \mathrm{~W} / \mathrm{mK}$. Neste exemplo, os resultados numéricos obtidos pela TVF, considerando a célula unitária com três fases (fibra, matriz e interfase), são comparados com aqueles apresentados em [14].

Tabela 1: Condutividade térmica das fibras e das interfases [14].

\begin{tabular}{l|l|l|l}
\hline TIPO DE FIBRA & $\begin{array}{l}\text { RAIO DA FIBRA } \\
(\boldsymbol{\mu m})\end{array}$ & $\begin{array}{l}\text { CONDUTIVIDADE TÉRMICA } \\
\text { DA FIBRA }(\mathbf{W} / \mathbf{m K})\end{array}$ & $\begin{array}{l}\text { CONDUTIVIDADE TÉRMICA } \\
\text { DA INTERFASE }(\mathbf{W} / \mathbf{m K})\end{array}$ \\
\hline Carbono & 3,5 & 11 & 5,595 \\
\hline Vidro & 10 & 1,3 & 0,745 \\
\hline
\end{tabular}

Para avaliar a influência da interfase sobre a condutividade térmica efetiva de compósitos, WANG e QIN [14] desenvolveram um elemento revestimento/fibra a partir do método híbrido de elementos finitos baseado em solução fundamental (HFS-FEM). Considerando a solução fundamental para fibra circular imersa em matriz infinita, o elemento criado dispensa a discretização do domínio da interfase e da fibra.

Como se observa na Figura 7, existe uma boa concordância entre os resultados encontrados pela TVF e por WANG e QIN [14] para ambos os tipos de fibras. Percebe-se que a condutividade efetiva aumenta juntamente com a espessura da interfase, visto que esta possui condutividade maior do que a matriz. O compósito com fibra de carbono apresentou um crescimento mais acentuado da condutividade efetiva com o aumento da espessura da interfase.

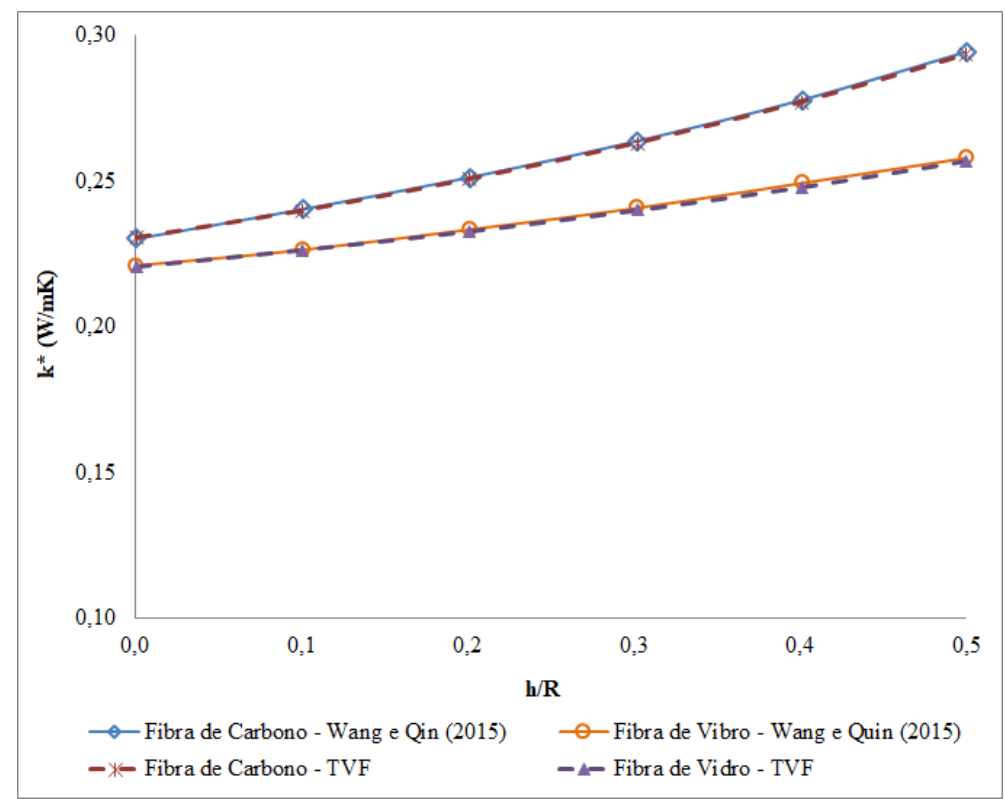

Figura 7: Condutividade térmica efetiva em função da espessura da interfase.

Também foi avaliada a influência da condutividade térmica da interfase $\mathrm{k}_{\mathrm{i}} \mathrm{e}$ de sua espessura. Para o compósito reforçado com fibras de carbono foram consideradas interfases com espessura de 0,35 $\mu \mathrm{m}, 0,70$ $\mu \mathrm{m}$ e $1,05 \mu \mathrm{m}$. Variando a relação $\mathrm{k}_{\mathrm{i}} / \mathrm{k}_{\mathrm{m}}$ de 0,001 a 1000, foram obtidos os resultados mostrados na Figura 8. 


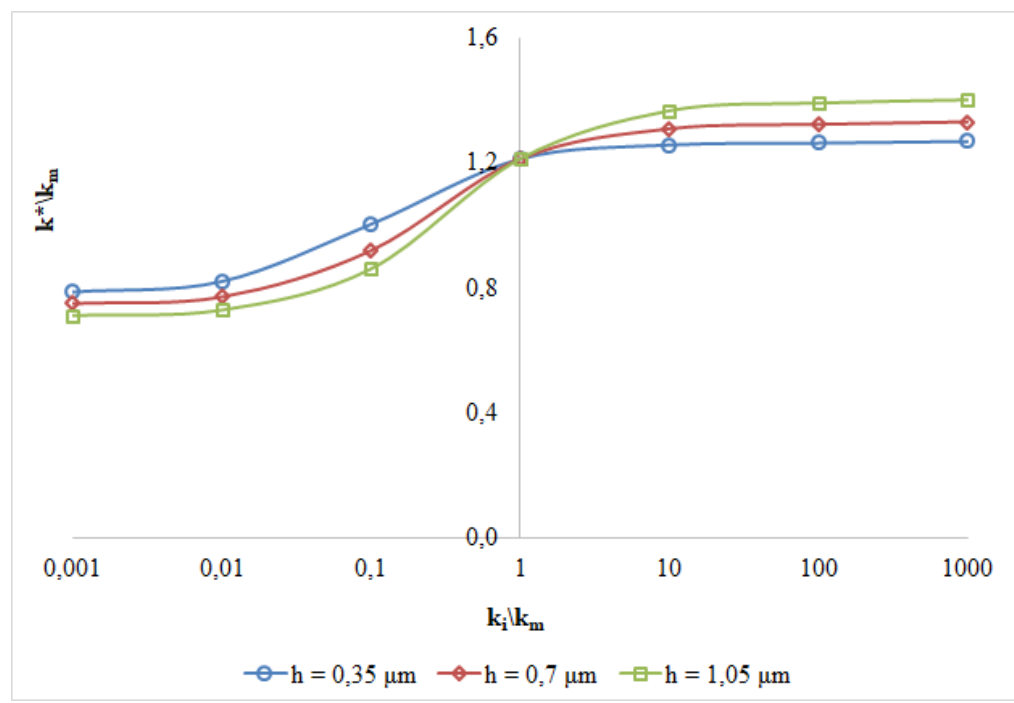

Figura 8: Compósito reforçado com fibra de carbono.

Para interfase com alta condutividade, ou seja, para $\mathrm{k}_{\mathrm{i}}$ variando de $10 \mathrm{k}_{\mathrm{m}}$ a $1000 \mathrm{k}_{\mathrm{m}}$, percebe-se que não ocorre aumento significativo de condutividade efetiva. Nessa faixa de valores de $\mathrm{k}_{\mathrm{i}}$, o acréscimo de condutividade térmica efetiva foi de aproximadamente $2,5 \%$ para a interfase mais espessa e de menos de $1 \%$ para a interfase mais fina. Comparando o caso de interfase altamente condutora com aquele sem interfase, ou seja, $\mathrm{k}_{\mathrm{i}}$ igual $\mathrm{k}_{\mathrm{m}}$, o compósito com interfase de espessura igual a 1,05 $\mu \mathrm{m}$ apresenta o maior acréscimo de condutividade efetiva, aproximadamente de $13 \%$. O compósito cuja interfase tem $0,7 \mu \mathrm{m}$ de espessura apresenta aumento de $9 \%$ na condutividade efetiva, aproximadamente o dobro do caso em que a interfase tem $0,35 \mu \mathrm{m}$.

Para o caso de interfase com baixa condutividade, alterações mais significativas na condutividade térmica efetiva são verificadas. O compósito cuja interfase possui $1,05 \mu \mathrm{m}$ de espessura e condutividade térmica igual a $0,1 \mathrm{k}_{\mathrm{m}}$ apresenta condutividade térmica efetiva $40 \%$ menor comparado com o compósito sem interfase. Para o compósito com interfase de $0,7 \mu \mathrm{m}$ de espessura, a condutividade efetiva diminui $32 \%$. Por sua vez, o compósito cuja interfase é igual a $0,35 \mu \mathrm{m}$ apresenta condutividade efetiva $20 \%$ menor. À medida que a condutividade térmica da interfase diminui, a diferença entre a condutividade térmica efetiva dos compósitos avaliados decresce. Esse fato demonstra que, para interfases muito pouco condutoras, a espessura das mesmas deixa de ser um fator importante, sendo mais preponderante o valor da condutividade da interfase.

Para o compósito reforçado com fibras de vidro foram avaliados os efeitos de interfases com espessuras de $1 \mu \mathrm{m}, 3 \mu \mathrm{m}$ e $5 \mu \mathrm{m}$. Variando-se a relação $\mathrm{k}_{\mathrm{i}} / \mathrm{k}_{\mathrm{m}}$ de 0,001 a 1000 , os resultados encontrados para estes casos estão mostrados na Figura 9.

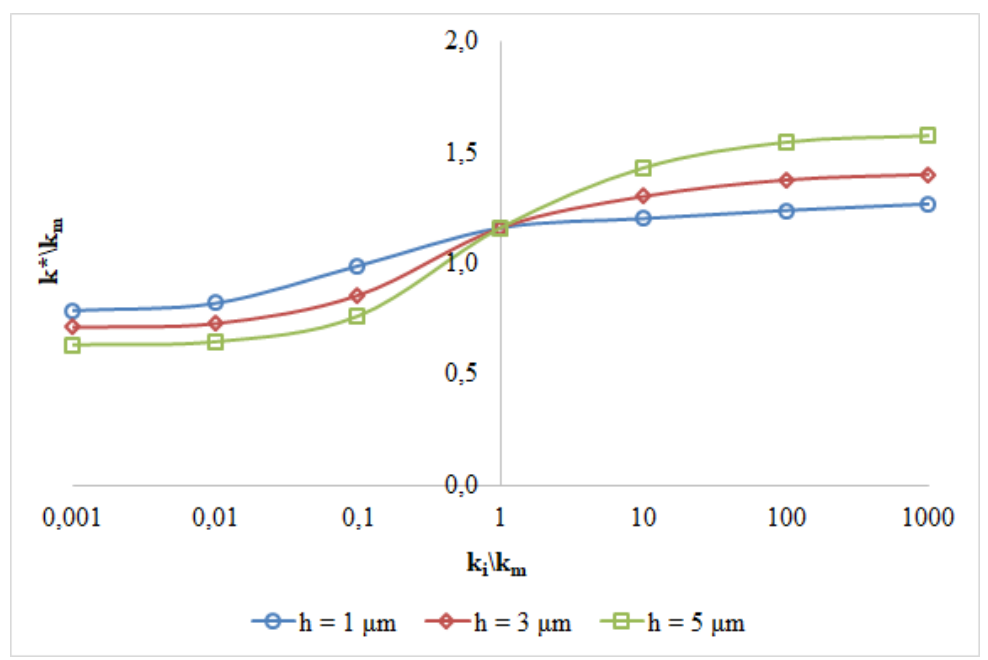

Figura 9: Compósito reforçado com fibra de vidro. 
O compósito com interfase de espessura igual a $5 \mu \mathrm{m}$ apresenta a maior variação de condutividade térmica efetiva quando a condutividade da interfase é modificada. Considerando interfases altamente condutivas, esse compósito apresenta um acréscimo de $26 \%$ na condutividade efetiva. Para o caso de interfase com baixa condutividade, o decréscimo é igual a $45 \%$. No caso do compósito cuja interfase tem $3 \mu \mathrm{m}$, a condutividade térmica efetiva aumenta em $18 \%$ quando $\mathrm{k}_{\mathrm{i}}=1000 \mathrm{k}_{\mathrm{m}}$ e diminui em $38 \%$ para $\mathrm{k}_{\mathrm{i}}=0,001 \mathrm{k}_{\mathrm{m}}$.

Por fim, o compósito com interfase de espessura igual a $1 \mu \mathrm{m}$ apresenta as menores variações na condutividade efetiva em comparação com o compósito sem interfase. Para o caso de interfase com baixa condutividade, o decréscimo na condutividade térmica efetiva é igual a 32\%. Para interfase altamente condutiva, a condutividade efetiva aumenta $8 \%$.

\subsection{Compósitos com fibras vegetais}

Neste exemplo é estudada a condutividade térmica efetiva transversal de compósitos reforçados por fibras vegetais unidirecionais. Os resultados numéricos obtidos pela TVF, considerando a célula unitária com três fases, são comparados com os resultados experimentais apresentados em [15].

As fibras vegetais são geralmente ocas, sendo os espaços vazios denominados lúmen. A Figura 10 ilustra um feixe de fibras vegetais, onde se destacam a região sólida e os lúmens.

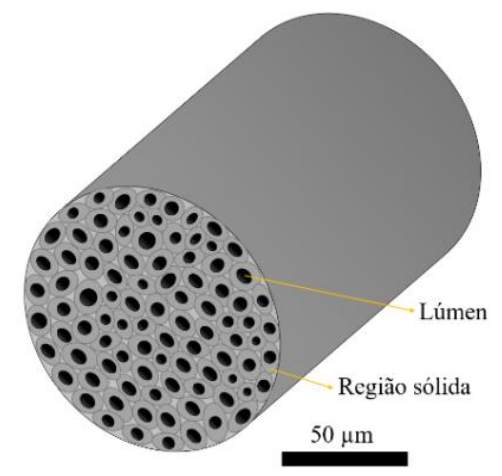

Figura 10: Feixe de fibras.

O compósito reforçado com fibras vegetais ocas pode ser tratado de forma aproximada como um compósito formado por três fases: matriz, fibra e lúmen. A Figura 11 mostra a seção transversal do compósito (a) e sua representação simplificada com distribuição periódica dos feixes de fibra (b). Desta distribuição periódica é escolhida uma célula de repetição unitária (c) cujo feixe de fibras apresenta diversos lúmens. Esta célula pode ser transformada em um modelo equivalente (d) onde os lúmens são agrupados, formando assim um modelo trifásico. 
(a)

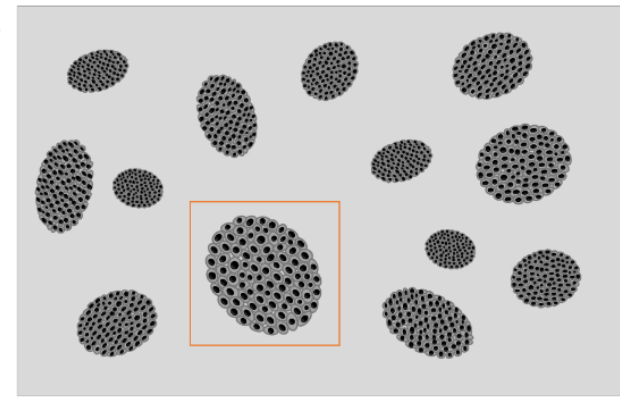

(d)

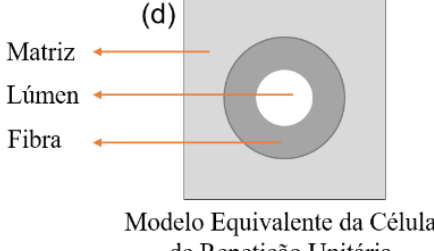

de Repetição Unitária

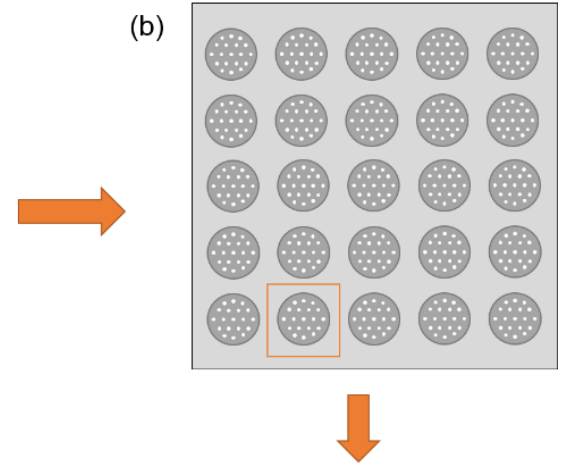

(c)

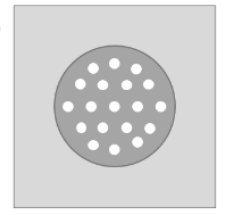

Célula de Repetição Unitária

Figura 11: Obtenção do modelo de três fases.

LIU et al. [15] determinaram experimentalmente a condutividade térmica de dois tipos de compósitos: um reforçado com fibras de abaca e outro com fibras de bambu, ambos com matriz epóxi. As condutividades térmicas da região sólida das fibras e da matriz estão apresentadas na Tabela 2. Maiores detalhes sobre as propriedades dos materiais podem ser obtidos em [15].

Tabela 2: Condutividade térmica dos materiais para o modelo trifásico.

\begin{tabular}{l|l}
\hline MATERIAL & $\begin{array}{l}\text { CONDUTIVIDADE } \\
\text { TÉRMICA (W/mK) }\end{array}$ \\
\hline Matriz epóxi & 0,298 \\
\hline Fibra de abaca & 0,430 \\
\hline Fibra de bambu & 0,350 \\
\hline Lúmen (ar) & 0,026 \\
\hline
\end{tabular}

Os feixes de fibras de abaca e bambu apresentam diferença significativa com relação à quantidade $\mathrm{e}$ tamanho do lúmen. A fração volumétrica de lúmen com relação ao feixe de fibras $\left(v_{l}\right)$ é representada por um coeficiente $\alpha$ [15], definido pela seguinte expressão:

$$
\alpha=\frac{r_{l}}{r_{f}}=\sqrt{\nu_{l}}
$$

Na Equação (18), $r_{l}$ e $r_{f}$ são os raios do lúmen e da região sólida, respectivamente. Os valores de $\alpha$ para as fibras de abaca e de bambu valem 0,67 e 0,12 , respectivamente.

Na Figura 12 são mostrados os resultados obtidos para o compósito reforçado com fibras de abaca, considerando frações volumétricas de feixe de fibras iguais a 13\%, 23\%, 34\% e 47\%. Neste caso, o raio da região sólida da fibra é igual a $93 \mu \mathrm{m}$ e o raio do lúmen vale $62 \mu \mathrm{m}$.

Como se observa na Figura 12, existe uma boa concordância entre os resultados numéricos e experimentais. A condutividade térmica efetiva do compósito diminui com o aumento da fração volumétrica de fibras. Esse comportamento se deve a presença do lúmen que atua como uma barreira para a transmissão de calor. 


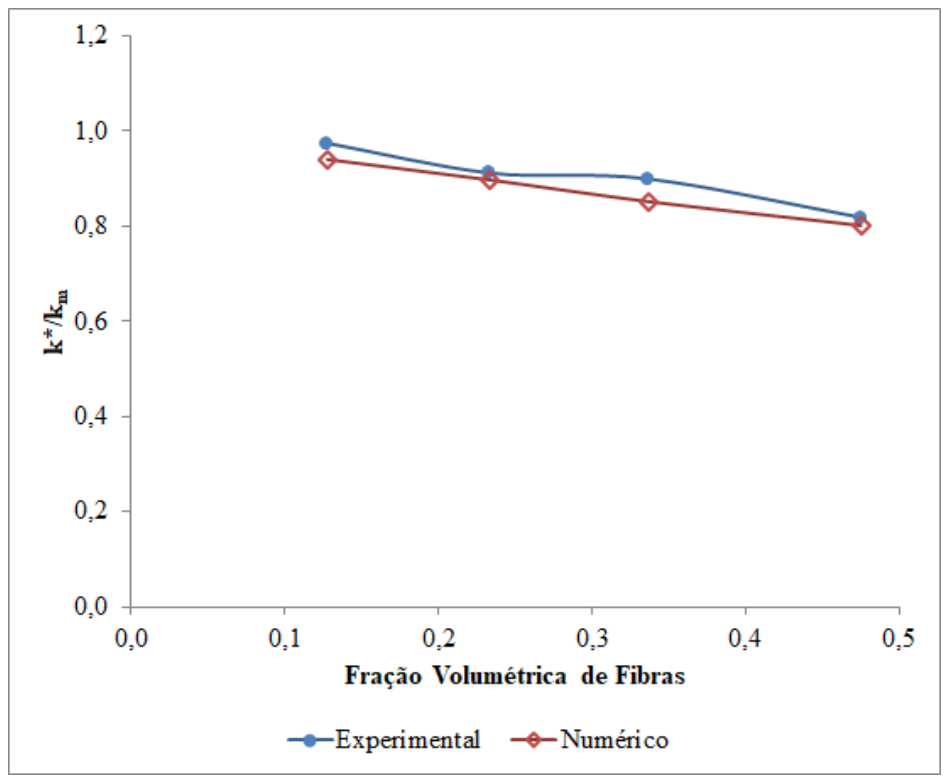

Figura 12: Condutividade térmica efetiva do compósito reforçado por fibras de abaca.

A Figura 13 apresenta os resultados obtidos para o compósito reforçado com fibras de bambu, considerando frações volumétricas de feixe de fibras iguais a 7\%, 14\% e 30\%. O raio da região sólida da fibra é igual a $102 \mu \mathrm{m}$ e o raio do lúmen vale $12,24 \mu \mathrm{m}$. Os resultados numéricos apresentam boa concordância com os resultados experimentais.

As dimensões do lúmen da fibra de bambu são pequenas, logo não influenciam de forma significativa a condutividade térmica efetiva do compósito. Dessa forma, há um pequeno acréscimo de condutividade com o aumento da fração volumétrica.

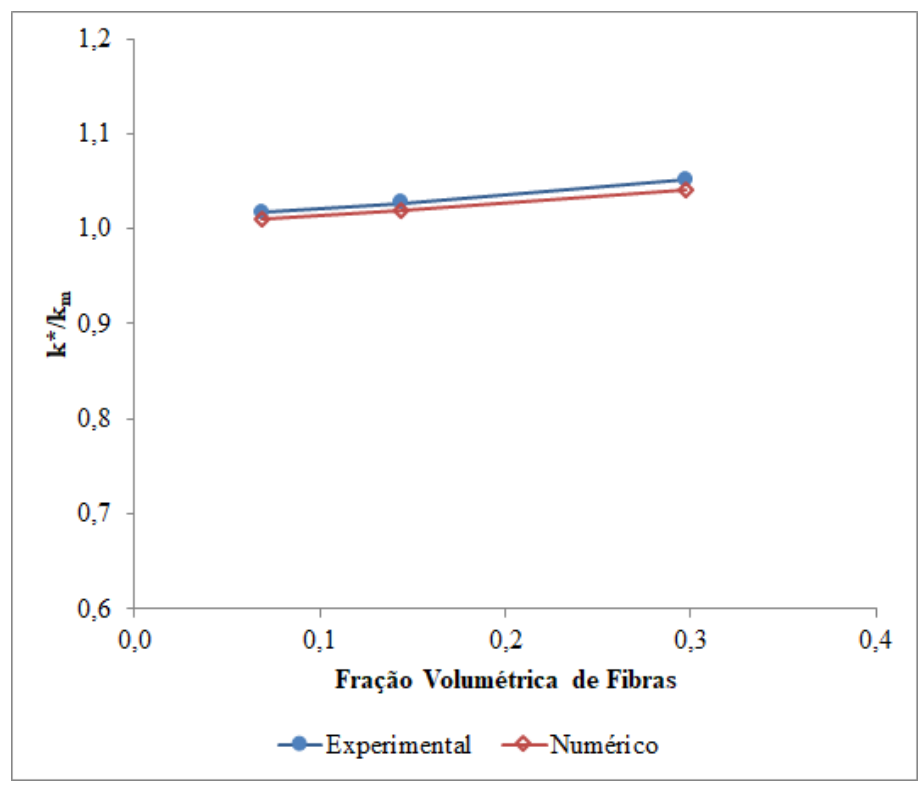

Figura 13: Condutividade térmica efetiva do compósito reforçado por fibras de bambu.

\section{CONCLUSÕES}

Neste trabalho foi avaliada a influência de interfases na condutividade térmica efetiva de compósitos periódicos reforçados por fibras unidirecionais. Verificou-se a influência da espessura da interfase e de sua condutividade, assim como o efeito de tamanho das fibras e de sua fração volumétrica. Também avaliou-se o efeito dos lúmens presentes em fibras vegetais. 
A condutividade térmica efetiva foi calculada pela formulação paramétrica da Teoria de Volumes Finitos utilizando células unitárias de repetição com três fases. Os resultados foram confrontados com os obtidos por outros métodos numéricos ou experimentos.

O crescimento da fração volumétrica implica em aumento da proporção interfase/matriz. Logo, quanto maior a fração volumétrica de fibras, maior é a influência da interfase na condutividade efetiva do compósito. Interfases pouco condutivas diminuem a condutividade térmica efetiva de acordo com a relação $\mathrm{k}_{\mathrm{i}} / \mathrm{k}_{\mathrm{m}}$. As interfases altamente condutivas proporcionam um aumento na condutividade efetiva, porém, esse aumento independe da relação $\mathrm{k}_{\mathrm{i}} / \mathrm{k}_{\mathrm{m}}$.

O efeito de tamanho da fibra se torna evidente quando se considera a presença da interfase. Mantendose a fração volumétrica constante, quanto maior o raio da fibra menor o volume total de interfase, diminuindo sua influência na condutividade efetiva. Dessa forma, fibras com raios menores apresentam condutividade térmica efetiva mais dependente da condutividade da interfase.

Quanto maior a espessura da interfase, maior sua influência na condutividade térmica efetiva, quando a fração volumétrica de fibras é mantida. Nos casos analisados, as interfases com baixa condutividade afetam mais drasticamente a condutividade efetiva do que as interfases altamente condutivas.

A condutividade térmica efetiva de compósitos com fibras vegetais depende do tamanho e da quantidade de lúmens presentes nas fibras. Dessa forma, conclui-se que os efeitos de interfase sobre o comportamento de compósitos são de extrema importância e, consequentemente, precisam ser considerados para proporcionar predições consistentes e realísticas.

\section{AGRADECIMENTOS}

Os autores agradecem o apoio financeiro proporcionado pelas agências CNPq, CAPES e FAPEAL.

\section{BIBLIOGRAFIA}

[1] GORI, F., CORASANITI, S., "Effective thermal conductivity of composites", International Journal of Heat and Mass Transfer, v. 77, p. 653-661, Oct. 2014.

[2] BURGER, N., LAACHACHI, A., FERRIOL, M., et al., "Review of thermal conductivity in composites: Mechanisms, parameters and theory”, Progress in Polymer Science, v. 61, p. 1-28, Oct. 2016.

[3] CHEN, H., GINZBURG, V.V., YANG, J., et al., "Thermal conductivity of polymer-based composites: fundamentals and applications", Progress in Polymer Science, v. 59, p. 41-85, Aug. 2016.

[4] LE QUANG, H., PHAN, T.-L., BONNET, G., "Effective thermal conductivity of periodic composites with highly conducting imperfect interfaces", International Journal of Thermal Sciences, v. 50, n. 8, p. 14281444, Aug. 2011.

[5] DASGUPTA, A., BHANDARKAR, S. M., “A generalized self-consistent Mori-Tanaka-Scheme for fibercomposites with multiple interphases", Mechanics of Materials, v. 14, n. 1, p. 67-82, Nov. 1992.

[6] BONFOH, N., SABAR, H., "Anisotropic thermal conductivity of composites with ellipsoidal inclusions and highly conducting interfaces”, International Journal of Heat and Mass Transfer, v. 118, p. 498-509, Mar. 2018.

[7] XIAO, J., XU, Y., ZHANG, F., "An analytical method for predicting the effective transverse thermal conductivity of nano coated fiber composites”, Composite Structures, v. 189, p. 553-559, Apr. 2018.

[8] PATHAK, S.V., Enhanced heat transfer in composite materials, Dissertação de M.Sc., Russ College of Engineering and Technology - Ohio University, OH, EUA, 2013.

[9] YANG, W., PENG, K., ZHOU, L., et al., "Finite element simulation and experimental investigation on thermal conductivity of diamond/aluminium composites with imperfect interface", Computational Materials Science, v. 83, p. 375-380, Feb. 2014.

[10] YVONNET, J., HE, Q.-C., TOULEMONDE, C., "Numerical modelling of the effective conductivities of composites with arbitrarily shaped inclusions and highly conducting interface", Composites Science and Technology, v. 68, n. 13, pp. 2818-2825, Oct. 2008.

[11] ESCARPINI FILHO, R.S., MARQUES, S.P.C., "A model for evaluation of effective thermal conductivity of periodic composites with poorly conducting interfaces", Materials Research, v. 17, n. 5, pp. 1344-135, Oct. 2014. 
[12] CAVALCANTE, M.A.A., MARQUES, S.P.C., PINDERA, M-J., "Parametric Formulation of the FiniteVolume Theory for Functionally Graded Materials. Part I: Analysis", ASME Journal of Applied Mechanics, v. 74, n. 5, p. 935-945, Sep. 2007.

[13] KAPITZA, P.L., “The study of heat transfer in helium II”, J. Phys., v. 4, pp. 181-210, 1941.

[14] WANG, H., QIN, Q-H., "A new special coating/fiber element for analyzing effect of interface on thermal conductivity of composites", Applied Mathematics and Computation, v. 268, pp. 311-321, Oct. 2015.

[15] LIU, K., TAKAGI, H., OSUGI, R., et al., "Effect of physicochemical structure of natural fiber on transverse thermal conductivity of unidirectional abaca/bamboo fiber composites", Composites: Part A, v. 43, n. 8 , p. 1234-1241, Aug. 2012.

\section{ORCID}

Camila de Sousa Vieira

Severino Pereira Cavalcanti Marques
https://orcid.org/0000-0002-5371-191X

https://orcid.org/0000-0003-3476-0289 